\title{
MEDICAL EMBEDDED SYSTEMS
}

\author{
Roozbeh Jafari, ${ }^{1}$ Soheil Ghiasi, ${ }^{2}$ and Majid Sarrafzadeh ${ }^{3}$ \\ ${ }^{1}$ Department of Electrical Engineering \\ University of Texas at Dallas \\ rjafari@utdallas.edu \\ ${ }^{2}$ Department of Electrical and Computer Engineering \\ University of California, Davis \\ soheil@ece.davis.edu \\ ${ }^{3}$ Department of Computer Science \\ University of California, Los Angeles \\ majid@cs.ucla.edu
}

\begin{abstract}
Light-weight embedded systems for medical monitoring are often referred to low-profile, small size, unobtrusive and potable processing elements with limited power resources. Such systems typically incorporate sensing, processing and communications and are often manufactured to be simple and cost-effective. Being low profile and wearable immediately implies the limitations in computational capabilities, memory (storage), speed and I/O interfaces. In this tutorial, we portray a brief description of low-power and light-weight embedded systems; present two pilot applications; and illustrate their corresponding design challenges. We specifically discuss the reconfiguration technqiues.
\end{abstract}

\section{INTRODUCTION}

Light-weight embedded systems are often referred to low-profile, small size, unobtrusive and potable processing elements with limited power resources. Such systems typically incorporate sensing, processing and communications and are often manufactured to be simple and cost-effective. Being low profile immediately implies the limitations in computational capabilities, memory (storage), speed and I/O interfaces. Despite their low complexity impedes the handling of computationally intensive tasks, they may be deployed in collaborative networks and potentially in large quantities. Their sensing capabilities allow their seamless integration with the physical world whilst their general- 
purpose architecture designs yield notable advantages such as reconfigurability and adaptability with various applications and environments. Consumers, on the other hand, constantly demand thinner, smaller and lighter systems with smaller batteries in which the battery life is enhanced to meet their lifestyle. Improving the performance of battery life, however, has been always a major scientific challenge for researchers. Several other key factors such as reliability, security, customizability and reconfigurability has been among the major concerns.

\section{TARGET APPLICATIONS}

\subsection{Physical Movement Monitoring}

Health care costs in developed countries are rapidly increasing due to a substantial increase the elderly population. Monitoring of daily physical activities can be a key to evaluating the actual quality of life among the elderly. We believe that the overall health and wellness of elderly sectors of the population can greatly benefit from the use of information and communication technology, especially for the homebound. Sensor platforms integrated into clothing provide the possibility of enhanced reliability of accident reporting and health monitoring. Such devices improve the independence of people needing living assistance [1]. In this tutorial, we outline a framework for movement assessment and classifying physical activities. In particular, we are interested in classifying transition movements for example, sit-to-stand, stand-to-sit, lie-tostand and stand-to-lie etc. The functionality of our automated pattern recognition system is divided into three basic tasks: the preprocessing and filtering, the description task which generates attributes of a movement using feature extraction techniques, and the classification task which classify this movement based on its attributes.

\subsection{Electrocardiogram Analysis}

The electrocardiogram (ECG) is a record of variation of bioelectric potential with respect to time as the human heart beats. Due to its ease of use and non-invasiveness, ECG plays an important role in patient monitoring and diagnosis. Multi-channel electrocardiogram (ECG) data provide cardiologists with essential information to diagnose heart disease in a patient. Our primary objective is to address the feasibility verification of implementing an ambulatory ECG analysis algorithm with real-time diagnosis functions for wearable computers. ECG analysis algorithms have always been very difficult tasks in the realization of computer aided ECG diagnosis. Implementation of such algorithms becomes even harder for small and mobile embedded systems that should meet the given latency requirements while minimizing overall energy 
dissipation for the system [2]. These obstacles may dramatically reduce the effectiveness of embedded distributed algorithms. Thus, a new distributed, embedded, computing attribute, dynamically reconfigurable, must be developed and provided to such systems. In these systems, reconfiguration capability, in particular, may be of great advantage. This capability can adaptively alter the system configuration to accommodate the objectives and meet the constraints for highly dynamic systems. Similar to movement monitoring, ECG analysis consists of preprocessing, pattern recognition, and classification.

\section{APPLICATION CHARACTERISTICS}

Most medical monitoring applications rely on pattern recognition and classification. Given the goal of classifying objects based on their attributes, the functionality of an automated pattern recognition system can be divided into two basic tasks: The description task generates attributes of an object using feature extraction techniques, and the classification task assigns a group label to the object based on the attributes with a classifier. There are two different approaches for implementing a pattern recognition system: statistical and structural. Each approach utilizes different schemes within the description and classification tasks which incorporates a pattern recognition system. Statistical pattern recognition [3][4] concludes from statistical decision theory to discriminate among data from different groups based upon quantitative features of the data. The quantitative nature of statistical pattern recognition, however, makes it difficult to discriminate among groups based on the morphological (i.e., shape-based or structural) sub-patterns and their interrelationships embedded within the data. This limitation provided the impetus for development of structural approaches to pattern recognition.

\section{RECONFIGURATION DESIGN TECHNIQUES}

Wearable health monitoring applications are characterized by their highly dynamic behavior, constrained hardware platform, critical missions, and potentially very large share in the marketplace. Consequently, the design process for such applications needs to be rethought to consider their specific requirements and platform challenges. Adaptive resource assignment and execution is one of the key requirements of medical applications. In this section, we present a number of situations that call for adaptive execution of target applications on medical embedded systems. We highlight several key research problems that will be discussed at length in our tutorial. These problems have to be addressed in order to develop adaptive resource assignment services. 


\subsection{High-Level Application Restructuring}

A unique characteristic of our target architecture is its ability in re-capturing sufficiently-correlated physical data from different body sensors. For example, heart beat signal can be sensed at a number of different locations on body. Therefore, a high-level application running on sufficiently sensor-populated wearable system that processes heart beat signal, has several choices for reading in the signal [2]. We illustrate high-level transformations that restructure a given application to take advantage of this specific application-dependent portunity. Example transformations include input re-capturing and task duplication. Besides input recapturing for sufficiently-correlated input data, replicating selected internal computations can create a favorable tradeoff between communication and computation demand, which is a great incentive to restructure the application at runtime.

\subsection{Dynamic Code Migration}

While some runtime events require new tasks to be invoked, another group of runtime events can be handled by partial restructuring of the application via task reallocation, without creation of new computations. For example, a newly launched application can overload a resource that is already executing a number of applications. In that case, we might be able to migrate some computing tasks, as well as the associated communications, to other resources to address the problem. Similarly, we can handle transient faults by reassignment of affected computations to other resources at price of degrading system performance, or increased energy dissipation.

\section{REFERENCES}

[1] Roozbeh Jafari, Wenchao Li, Ruzena Bajcsy, Steven Glaser and Shankar Sastry. "Physical Activity Monitoring for Assisted Living at Home". In International Workshop on Wearable and Implantable Body Sensor Networks (BSN), March 2007.

[2] Roozbeh Jafari, Hyduke Noshadi, Soheil Ghiasi and Majid Sarrafzadeh. "Adaptive Electrocardiogram Feature Extraction on Distributed Embedded Systems". IEEE Transactions on Parallel and Distributed Systems Special Issue on High Performance Computational Biology, 17(8):797-807, Aug 2006.

[3] Richard O. Duda, Peter E. Hart, and David G. Stork. Pattern Classification. John Wiley and Sons, Inc., second edition, 2000.

[4] Anil K. Jain, Robert P. W. Duin, and Jianchang Mao. Statistical pattern recognition: A review. IEEE Trans. Pattern Anal. Mach. Intell., 22(1):4-37, 2000. 